\title{
Verhaltenskodex für Berufsorganisationen des Gesundheitswesens im Kampf gegen das Rauchen
}

\author{
Verabschiedet und unterzeichnet von den Teilnehmern der informellen Konferenz der WHO über die Gesundheitsberufe \\ und den Kampf gegen das Rauchen. Genf, 28.-30. Januar 2004
}

\section{Einleitung}

Um einen aktiven Beitrag zur Einschränkung des Tabakkonsums zu leisten und um dafür zu sorgen, dass der Kampf gegen das Rauchen ein Anliegen des öffentlichen Gesundheitswesens auf regionaler, nationaler und internationaler Ebene wird, werden im vorliegenden Kodex folgende Richtlinien für die Berufsverbände im Gesundheitswesen festgelegt:

1. Sie ermutigen und unterstützen ihre Mitglieder, auf das Rauchen zu verzichten und eine Lebenskultur ohne Rauchen zu fördern.

2. Sie evaluieren und untersuchen mit Hilfe von Meinungsumfragen und mit dem Einsatz geeigneter Mittel die verschiedenen Arten des Tabakkonsums sowie die Einstellung und das Verhalten ihrer Mitglieder im Kampf gegen das Rauchen.

3. Sie setzen sich bei Kongressen und Konferenzen zu diesem Thema für die Propagierung des Nichtrauchens ein.

4. Sie setzen den Kampf gegen das Rauchen auf die Tagesordnung aller Kongresse und Konferenzen zum Thema Gesundheit.

5. Sie empfehlen ihren Mitgliedern, systematisch ihre Patienten und Kunden zu befragen, ob sie rauchen oder ob sie Tabakrauch ausgesetzt sind. Dabei informieren sie über wissenschaftliche und medizinische sowie praktische Erfahrungen und Methoden, mit dem Rauchen aufzuhören und bieten ihnen geeignete Hilfestellung und Betreuung während der Entzugsphase.

6. Sie fordern Gesundheitseinrichtungen und medizinische Ausbildungsstätten dazu auf, den Kampf gegen das Rauchen durch Weiter- bildung und verschiedene Schulungs- und Seminarangebote in die Ausbildung zu Heilberufen zu integrieren.

7. Sie nehmen jedes Jahr am 31. Mai aktiv am Welt-Nichtrauchertag teil.

8. Sie leisten der Tabakindustrie keinerlei finanzielle oder sonstige Unterstützung, investieren nicht in die Tabakindustrie und ermutigen ihre Mitglieder, ihrem Beispiel zu folgen.

9. Sie vergewissern sich, dass ihre Organisation eine klare Politik in Geschäftsbeziehungen bzw. allen anderen Beziehungen zu Partnern verfolgt, von denen bekannt ist, dass sie über Beziehungen oder Beteiligungen in der Tabakindustrie verfügen. Sie lassen sich von diesen Partnern eine Interessenerklärung unterzeichnen.

10. Sie ermutigen Institutionen, Verkauf, Werbung oder Kauf von Tabakwaren in ihrem Bereich zu verbieten, und fordern ihre Mitglieder auf, dasselbe zu tun.

11. Sie leisten den Regierungen in dem Prozess, der zu Unterzeichnung, Ratifizierung und Durchführung des WHO-Rahmenabkommens zum Kampf gegen das Rauchen führen soll, aktive Unterstützung.

12. Sie stellen finanzielle und andere Mittel für den Kampf gegen das Rauchen zur Verfügung, wie auch für die Einsetzung und Befolgung des vorliegenden Verhaltenskodexes.

13. Sie nehmen aktiv an von Berufsverbänden des Gesundheitswesens veranstalteten Antiraucherkampagnen teil.

14. Sie unterstützen Kampagnen, die sich für das Rauchverbot an öffentlichen Orten einsetzen. 